\title{
Semi-Analytic Solution of HIV and TB Co-Infection Model
}

\section{${ }^{* 1}$ BOLARIN, G; ${ }^{2}$ OMATOLA, IU; ${ }^{3}$ AIYESIMI, YM; ${ }^{4}$ YUSUF, A}

\author{
${ }^{1,2,3,4}$ Department of Mathematics, Federal University of Technology, Minna, 23401, Nigeria \\ I'g.bolarin@futminna.edu.ng, ${ }^{2}$ innocentukwubile@gmail.com, ${ }^{3}$ yomi_aiyesimi2007@yahoo.co.uk, ${ }^{4}$ yusuf.abdulhakeem@futminna.edu.ng
}

Author for Correspondence: g.bolarin@futminna.edu.ng, +23408187141978

\begin{abstract}
In this work we developed and analyzed a mathematical model of HIV and TB coinfection. The model is a first order Ordinary Differential Equations, in which the human population is divided into six mutually- exclusive compartments namely; TB- Susceptible individuals (S), TB-Infected individuals (I), TB-Recovered individuals (R), HIV-Infected individuals $\left(\mathrm{P}_{1}\right)$, Co- Infected individuals $\left(\mathrm{P}_{2}\right)$ and individuals with AIDS (A). The analytical solution is obtained using Homotopy Perturbation Method (HPM). The result of the numerical simulation shows that at high HIV and TB treatment rates; TB, AIDS and HIV/TB co-infection will be eradicated completely in the population. Also, early detection of HIV and TB cases and provision of early treatments can reduce the rate of infection, reduce the rate of progression of HIV infected individuals to AIDS and lowers co-infection. @JASEM
\end{abstract}

https://dx.doi.org/10.4314/jasem.v21i2.7

Keywords: Tuberculosis, Human Immunodeficiency Virus, Co-infection, Treatment and Homotopy Perturbation Method (HPM).

Tuberculosis (TB) and Human Immunodeficiency Virus/Acquired Immune Deficiency Syndrome (HIV/AIDS) constitute the main burden of infectious disease in resource-limited countries. Estimates by the World Health Organization (WHO) indicate that there are more than 9 million new active cases of TB and close to 2 million deaths per year WHO (2010). In the individual host the two pathogens, M. tuberculosis and HIV, potentiate one another, accelerating the deterioration of immunological functions and resulting in premature death if left untreated. About 14 million individuals worldwide are estimated to be dually infected. Both TB and HIV have profound effects on the immune system, as they are capable of disarming the host's immune responses through mechanisms that are not fully understood. HIV/TB co-infection is the most powerful known risk factor for progression of $\mathrm{M}$. tuberculosis infection to active disease, increasing the risk of latent TB reactivation 20-fold Getahun et al., (2010). Bolarin, 2012; in his work, formulated a sex-structured model to capture the effect of complacency on the dynamics of HIV/AIDS but did not include how TB will affect the mix. But, recently a lot of ground work has been covered in the mathematical modelling of coinfection of different pathogens though very little was done in the modelling of HIV/TB co infection. David, 2013; Nthiiri, 2015; Roeger et al., (2009), Shah and Jyota (2013) have developed mathematical models of HIV/TB co-infection under TB treatment. Their work did not include anti-HIV treatment. Silver and Delfim (2015) developed a TB-HIV/AIDS co-infection model and optimal control treatment.

One of the most powerful methods to approximately solve linear and non-linear differential equations is the Homotopy Perturbation Method (HPM); see Abubakar et al., (2013), (Jiya, 2010) for examples. The HPM method is based in the use of a power series, which transforms the original non-linear differential equation into a series of linear differential equations. Two continuous functions from one topological space to another are called homotopic if one can be "continuously deformed" into the other, such a deformation is called a homotopy between the two functions. The Homotopy Perturbation Method (HPM), which provides analytical approximate solution, is applied to various linear and non-linear equations (He, 1999). The Homotopy Perturbation Method (HPM) is a series expansion method used in the solution of non-linear partial differential equations (Jiya, 2010). The method employs a 
homotopy transform to generate a convergent series solution of differential equations. To illustrate the basic ideas of this method, the following non-linear differential equation was considered (He, 2000).

$B\left(U, \frac{\partial U}{\partial n}\right)=0 r \in \Gamma$

$A(u)-f(r)=0, r \in \Omega$,

Subject to the boundary condition

Where $\mathrm{A}$ is a general differential operator, B a boundary operator, $f(r)$ is a known analytical function and $\Gamma$ is the boundary of the domain $\Omega$. The operator A can be divided into two parts $\mathrm{L}$ and $\mathrm{N}$, where $\mathrm{L}$ is the linear part, and $\mathrm{N}$ is the nonlinear component. Equation (1) may therefore be written as:

$L(U)+N(U)-f(r)=0, \quad r \in \Omega$

The Homotopy Perturbation structure is shown as follows;

$H(V, h)=(1-h)\left[L(V)-L\left(U_{0}\right)\right]+h[A(V)-f(r)]=0$

Where $V(r, P): \Omega \in[0,1] \rightarrow R$

In equation (3) $P \in[0,1]$ is an embedding parameter and $U_{0}$ is the approximation that satisfies the boundary condition. It can be assumed that the solution of the equation (3) can be

Written as power series in $\mathrm{h}$ as follows:

$V=V_{0}+h V_{1}+h^{2} V_{2}+\ldots$

And the best approximation for the solution is:

$U=\lim _{h \rightarrow 1} v=v_{0}+h v_{1}+h^{2} v_{2}+\ldots$

The series (6) is convergent for most cases. However, the convergent rate depends on the nonlinear operator A (V)

The derivation and analysis of the HIV/TB co-infection model used in this work can be found in Bolarin and Omatola (2016). The main objective of this project is to develop a mathematical model for control and elimination of HIV and TB Co-Infection in the presence of HIV and TB treatments.

\section{MATERIALS AND METHODS}

\section{HIV/TB Model}

$\frac{d s}{d t}=\Lambda+r R-\left(\lambda_{T}+\lambda_{H}+\mu\right) S$

$\frac{d I}{d t}=\lambda_{T} S-\left(\lambda_{H}+a+d_{T}+\mu\right) I$

BOLARIN, G.; OMATOLA, I.U.; AIYESIMI, Y.M. and YUSUF, A. 


$$
\begin{aligned}
& \frac{d R}{d t}=a I-\left(\lambda_{H}+r+\mu\right) R \\
& \frac{d P_{1}}{d t}=(S+R) \lambda_{H}+\left(b_{1}+b_{2}\right) P_{2}-\left(\lambda_{T}+e+d_{H}+\mu\right) P_{1} \\
& \frac{d P_{2}}{d t}=\lambda_{H} I+\lambda_{T} P_{1}-\left(b_{1}+b_{2}+d+d_{H 1}+\mu\right) P_{2} \\
& \frac{d A}{d t}=e P_{1}+d P_{2}-\left(d_{A}+\mu\right) A
\end{aligned}
$$

\section{Where}

$\mathrm{N}=\mathrm{S}+\mathrm{I}+\mathrm{R}+\mathrm{P}_{1}+\mathrm{P}_{2}+\mathrm{A}$

$\mathrm{N} \quad$ total population

$\mathrm{S} \quad$ number of susceptible (that is no infection)

I number of persons with active TB

$\mathrm{R}$ number of persons recovered from TB

$\mathrm{P}_{1} \quad$ number of persons with HIV infection

$\mathrm{P}_{2} \quad$ number of persons with both HIV and TB infection

A number of persons with AIDS

W number of active population

Constant recruitment rate

$\beta_{T} \quad$ Probability of transmission of TB infection from an active to a susceptible Per contact per unit time

$\beta_{H} \quad$ Probability of transmission of HIV infection from an infected person to

an uninfected person per contact per unit time per capita contact rate for TB per capita contact rate for HIV Natural death rate treatment rate of active TB individuals treatment rate of infectious TB in HIV individuals HIV treatment rate AIDS progression rate for individuals in $\mathrm{P}_{2}$ AIDS progression rate for individuals in $\mathrm{P}_{1}$ rate at which $\mathrm{TB}$ recovered individuals become susceptible to TB active TB induced death rate

$\mathrm{d}_{\mathrm{H} 1}$ death rate due to both HIV and TB infection

$\mathrm{d}_{\mathrm{H}} \quad$ HIV induced death rate 
$\mathrm{d}_{\mathrm{A}}$ AIDS induced death rate

\section{Solution of the Model Equations}

$$
\begin{aligned}
& \frac{d S}{d t}+\left(\lambda_{T}+\lambda_{H}+\mu\right) S-r R-\Lambda=0 \\
& \frac{d I}{d t}+\left(\lambda_{H}+a+d_{T}+\mu\right) S-\lambda_{T} S=0 \\
& \frac{d R}{d t}+\left(\lambda_{H}+r+\mu\right) R-a I=0 \\
& \frac{d P_{1}}{d t}+\left(\lambda_{T}+e+d_{H}+\mu\right) P_{1}-(S+R) \lambda_{H}-\left(b_{1}+b_{2}\right) P_{2}=0 \\
& \frac{d P_{2}}{d t}+\left(b_{1}+b_{2}+d+d_{H 1}+\mu\right) P_{2}-\lambda_{H} I-\lambda_{T} P_{1}=0 \\
& \frac{d A}{d t}+\left(d_{A}+\mu\right) A-e P_{1}-d P_{2}=0
\end{aligned}
$$

With the following initial conditions $S(0)=S_{0}, I(0)=I_{0}, R(0)=R_{0}, P_{1}(0)=P_{1} o$,

$$
P_{2}(0)=P_{2} o \text { And } A(0)=A_{0}
$$

Let

$S=c_{0}+h c_{1}+h^{2} c_{2}+\ldots$

$I=e_{0}+h e_{1}+h^{2} e_{2}+\ldots$

$R=w_{0}+h w_{1}+h^{2} w_{2}+\ldots$

$P_{1}=x_{0}+h x_{1}+h^{2} x_{2}+\ldots$

$P_{2}=y_{0}+h y_{1}+h^{2} y_{2}+\ldots$

$A=z_{0}+h z_{1}+h^{2} z_{2}+\ldots$

Applying HPM to (13)

$(1-h) \frac{d S}{d t}+h\left[\frac{d S}{d t}+\left(\lambda_{T}+\lambda_{H}+\mu\right) S-r R-\Lambda\right]=0$

Substituting equations (19) and (21) into equation (25)

$$
\begin{aligned}
& (1-h)\left(c_{0}^{\prime}+h c_{1}^{\prime}+h^{2} c_{2}^{\prime}+\ldots\right) \\
& +h\left[\begin{array}{l}
\left(c_{0}^{\prime}+h c_{1}^{\prime}+h^{2} c_{2}^{\prime}+\ldots\right)+\left(\lambda_{T}+\lambda_{H}+\mu\right)\left(c_{0}+h c_{1}+h^{2}+\ldots\right) \\
-r\left(w_{0}+h w_{1}+h^{2} w_{2}+\ldots\right)-\Lambda
\end{array}\right]=0
\end{aligned}
$$

BOLARIN, G.; OMATOLA, I.U.; AIYESIMI, Y.M. and YUSUF, A. 
Expanding and collecting the coefficients of the powers of $h$, we have

$h^{0}: c_{1}^{i}=0$

$$
h^{\prime}: c_{1}^{\prime}+\left(\lambda_{T}+\lambda_{H}+\mu\right) c_{0}-r w_{0}-\Lambda=0
$$

$h^{2}: c_{2}^{\prime}+\left(\lambda_{T}+\lambda_{H}+\mu\right) c_{1}-r w_{1}=0$

$$
h^{3}:\left(\lambda_{T}+\lambda_{H}+\mu\right) c_{2}-r w_{2}=0
$$

Applying (H P M) to (14)

$(1-h) \frac{d I}{d t}+h\left[\frac{d I}{d t}+\left(\lambda_{H}+a+d_{T}+\mu\right) I-\lambda_{T} S\right]=0$

Substitute equation (19) and (20) into equation (31)

$e_{0}^{\prime}+h e_{1}^{\prime}+h^{2} e_{2}^{\prime}+\ldots$

$+h\left[\begin{array}{l}\left(\lambda_{H}+a+d_{T}+\mu\right)\left(e_{0}+h e_{1}+h^{2} e_{2}+\ldots\right) \\ -\lambda_{T}\left(c_{0}+h c_{1}+h^{2} c_{2}+\ldots\right)\end{array}\right]$

Collecting the coefficient of the powers of $h$, we have

$h^{0}: e_{0}^{\prime}=0$

$h^{1}: e_{1}^{\prime}+\left(\lambda_{H}+a+d_{T}+\mu\right) e_{0}-\lambda_{T} c_{0}=0$

$h^{2}: e_{2}^{\prime}+\left(\lambda_{H}+a+d_{T}+\mu\right) e_{1}-\lambda_{T} c_{1}=0$

$h^{3}:\left(\lambda_{H}+a+d_{T}+\mu\right) e_{2}-\lambda_{T} c_{2}=0$

Applying H P M to (15)

$(1-h) \frac{d R}{d t}+h\left[\frac{d R}{d t}+\left(\lambda_{H}+r+\mu\right) R-a I\right]=0$

Substitute equation (20) and (21) into equation (37)

$(1-h)\left(\dot{w}_{0}+h \dot{w}_{1}+h^{2} w_{2}+. ..\right)$

$+h\left[\begin{array}{l}\left(w_{0}+h \dot{w}_{1}+h^{2} \dot{w}_{2}+\ldots\right)+\left(\lambda_{H}+r+\mu\right)\left(w_{0}+h w_{1}+h^{2} w_{2}+. .\right) \\ -a\left(e_{0}+h e_{1}+h^{2} e_{2}+\ldots\right)\end{array}\right]=0$

Expanding and collecting the coefficients of the power of h, we have

$h^{0}: w_{0}^{\prime}=0$

BOLARIN, G.; OMATOLA, I.U.; AIYESIMI, Y.M. and YUSUF, A. 
$h^{1}: w_{1}^{\prime}+\left(\lambda_{H}+r+\mu\right) w_{0}-a e_{0}=0$

$h^{2}: w_{2}^{\prime}+\left(\lambda_{H}+r+\mu\right) w_{1}-a e_{1}=0$

$h^{3}:\left(\lambda_{H}+r+\mu\right) w_{2}-a e_{2}=0$

Applying HPM to (16)

$\frac{d P_{1}}{d t}+\left(\lambda_{T}+e+d_{H}+\mu\right) P_{1}-(S+R) \lambda_{H}-\left(b_{1}+b_{2}\right) P_{2}=0$

Substitute equation (19), (21), (22) and (23) into equation (43)

$(1-h)\left(\dot{x}_{0}^{\prime}+h \dot{x}_{1}^{\prime}+h^{2} \dot{x}_{2}^{\prime}+\ldots\right)$

$h\left[\begin{array}{l}\left(x_{0}^{\prime}+h x_{1}^{\prime}+h^{2} x_{2}^{\prime}+\ldots\right) \\ +\left(\lambda_{T}+e+d_{H}+\mu\right)\left(x_{0}+h x_{1}+h^{2} x_{2}+\ldots\right) \\ -\left(c_{0}+h c_{1}+h^{2} c_{2}+\ldots+w_{0}+h w_{1}+h^{2} w_{2}\right) \lambda_{H}-b\left(y_{0}+h y_{1}+h^{2} y_{2}+\ldots\right)\end{array}\right]=0$

Collecting the coefficients of the powers of $h$

$h^{0}: x_{0}^{\prime}=0$

$h^{1}: x_{1}^{\prime}+\left(\lambda_{T}+e+d_{H}+\mu\right) x_{0}-\left(c_{0}+w_{0}\right) \lambda_{H}-b y_{0}$

$h^{2}: x_{2}^{\prime}+\left(\lambda_{T}+e+d_{H}+\mu\right) x_{1}-\left(c_{1}+w_{1}\right) \lambda_{H}-b y_{1}$

$h^{3}:\left(\lambda_{T}+e+d_{H}+\mu\right) x_{2}-\left(c_{2}+w_{2}\right) \lambda_{H}-b y_{2}$

Applying HPM to (17)

$(1-h) \frac{d P_{2}}{d t}+h\left[\frac{d P_{2}}{d t}+\left(b_{1}+b_{2}+d+d H_{1}+\mu\right) P_{2}-\lambda_{H} I-\lambda_{T} P_{1}\right]=0$

Substitute equations (20), (22) and (23) into equation (49)

$$
\begin{aligned}
& (1-h)\left(y_{0}^{\prime}+h y_{1}^{\prime}+h^{2} y_{2}^{\prime}+\ldots\right) \\
& +h\left[\begin{array}{l}
\left(y_{0}^{\prime}+h y_{1}^{\prime}+h^{2} y_{2}^{\prime}+\ldots\right)+\left(b_{1}+b_{2}+d+d_{H}+\mu\right)\left(y_{0}+h y_{1}+h^{2} y_{2}+\ldots\right) \\
-\left(e_{0}+h e_{1}+h^{2} e_{2}+\ldots\right) \lambda_{H}-\lambda_{T}\left(j_{0}+h j_{1}+h^{2} j_{2}+\right)
\end{array}\right]=0
\end{aligned}
$$

Collecting the coefficient of the powers of $h$

$h^{0}: y_{0}^{\prime}=0$

$h^{\prime}: y_{1}^{\prime}+\left(b_{1}+b_{2}+d+d_{H 1}+\mu\right) y_{0}-e_{0} \lambda_{H}-x_{0} \lambda_{T}$

BOLARIN, G.; OMATOLA, I.U.; AIYESIMI, Y.M. and YUSUF, A. 
$h^{2}: y_{2}^{\prime}+\left(b_{1}+b_{2}+d+d_{H 1}+\mu\right) y_{1}-e_{1} \lambda_{H}-x \lambda_{T}$

$$
h^{3}:\left(b+b_{2}+d+d_{H 1}+\mu\right) y_{2}-e_{2} \lambda_{H}-x_{2} \lambda_{T}
$$

Applying HPM to (18)

$(1-h) \frac{d A}{d t}+h\left[\frac{d A}{d t}+\left(d_{A}+\mu\right) A-e P_{1}-d P_{2}\right]=0$

Substitute equation (22), (23) and (24) into equation (55)

$(1-h)\left(z_{0}+h z_{1}^{\prime}+h^{2} z_{2}^{1}+\ldots\right)$
$+h\left[\begin{array}{l}\left(z_{0}+h z_{1}+h^{2} z_{2}^{1}+\ldots\right) \\ +\left(d_{A}+\mu\right)\left(z_{0}+h z_{1}+h^{2} z_{2}+\ldots\right) \\ -e\left(x_{0}+h x_{1}+h^{2} x_{2}+\ldots\right) \\ -d\left(y_{0}+h y_{1}+h^{2} y_{2}+\ldots\right)\end{array}\right]=0$

Collecting the coefficients of the powers of $h$,

We have

$h^{0}: z_{0}^{\prime}=0$

$h^{1}: z_{1}^{\prime}+\left(d_{A}+\mu\right) z_{0}-e x_{0}-d y_{0}=0$

$h^{2}: z_{2}^{\prime}+\left(d_{A}+\mu\right) z_{1}-e x_{1}-d y_{1}=0$

$h^{3}:\left(d_{A}+\mu\right) z_{2}-e x_{2}-d y_{2}=0$

Solving equations (27), (28), (29) and (30) we have:

$S(t)=S_{0}+\left(r R_{0}+\Lambda-\left(\lambda_{T}+\lambda_{H}+\mu\right) S_{0}\right) t$

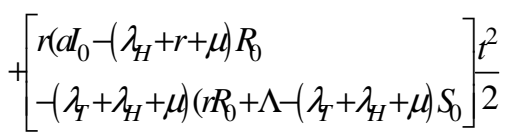

Solving equations (33), (34), (35) and (36) we have:

$$
\begin{aligned}
& I(t)=I_{0}+\left(\lambda_{T} S_{0}-\left(\lambda_{H}+a+d_{T}+\mu\right) I_{0}\right) t \\
& \left.+\left[\begin{array}{l}
\lambda_{T}\left(r R_{0}+\Lambda-\left(\lambda_{H}+\lambda_{T} \mu\right) S_{0}\right)- \\
\left(\lambda_{H}+a+d_{T}+\mu\right)\left(\lambda_{T} S_{0}-\left(\lambda_{H}+a+d_{T}+\mu\right) I_{0}\right)
\end{array}\right]\right) \frac{t^{2}}{2}
\end{aligned}
$$

Solving equations (39), (40), (41) and (42) we have:

BOLARIN, G.; OMATOLA, I.U.; AIYESIMI, Y.M. and YUSUF, A. 
$R(t)=R_{0}+\left(a I_{0}-\left(\lambda_{H}+r+\mu\right) R_{0}\right) t+\left[a\left(\lambda_{T} S_{0}-\left(\lambda_{H}+a+d_{T}+\mu\right) I_{0}\right)\right.$

$\left.-\left(\lambda_{H}+r+\mu\right)\left(a I_{0}-\left(\lambda_{H}+r+\mu\right) R_{0}\right)\right] \frac{t^{2}}{t}$

Solving equations (45), (46), (47) and (48) we have:

$P_{1}(t)=P_{1} o+\left(\left(S_{0}+R_{0}\right) \lambda_{H}+\left(b_{1}+b_{2}\right) P_{2} o-\left(\lambda_{T}+e+d_{H}+\mu\right) P_{1} o\right) t+$

$\left[\begin{array}{l}\left(r R_{0}+\Lambda-\left(\lambda_{T}+\lambda_{H}+\mu\right) S_{0}+\left(a I_{0}-\left(\lambda_{H}+r+\mu\right) R_{0}\right) \lambda_{H}\right. \\ +\left(b_{1}+b_{2}\right)\left(I_{0} \lambda_{H}+P_{1} o \lambda_{T}-\left(b_{1}+b_{2}+d+d_{H 1}+\mu\right) P_{2} o\right) \\ -\left(\lambda_{T}+e+d_{H}+\mu\right)\left(\left(S_{0}+R_{0}\right) \lambda_{H}+\right. \\ \left.\left(b_{1}+b_{2}\right) P_{2} o-\left(\lambda_{T}+e+d_{H}+\mu\right) P_{1} o\right)\end{array}\right] \frac{t^{2}}{2}$

Solving equations (51), (52), (53) and (54) we have:

$P_{2}(t)=P_{2} o+\left(I_{0} \lambda_{H}+P_{1} o \lambda_{T}-\left(b_{1}+b_{2}+d+d_{H 1}+\mu\right) P_{2} o\right) t$

$+\left[\left(\lambda_{T} S_{0}-\left(\lambda_{H}+a+d_{T}+\mu\right) I_{0}\right) \lambda_{H}+\lambda_{H}\left(\left(S_{0}+R_{0}\right) \lambda_{H}\right.\right.$

$\left.+\left(b_{1}+b_{2}\right) P_{2} o\right]-\left(\lambda_{T}+e+d_{H}+\mu\right) P_{1} o-\left(b_{1}+b_{2}+d+d_{H 1}+\mu\right)$

$\left.\left(I_{0} \lambda_{H}+P_{1} o \lambda_{T}-\left(b_{1}+b_{2}+d+d_{H 1}+\mu\right) P_{2} o\right)\right] \frac{t^{2}}{2}$

Solving equations (57), (58), (59) and (60) we have:

$A(t)=A_{0}+\left(e P_{1} o+d P_{2} o-\left(d_{A}+\mu\right) A_{0}\right) t+$

$\left[e\left(\left(S_{0}+R_{0}\right) \lambda_{H}+b P_{2} o-\left(\lambda_{T}+e+d_{H}+\mu\right) P_{1} o\right)+\right.$

$d\left(I_{0} \lambda_{T}+P_{1} o \lambda_{T}-\left(b+d+d_{H 1}+\mu\right) P_{2} o\right)$

$\left.-\left(d_{A}+\mu\right)\left(e P_{1} o+d P_{2} o-\left(d_{A}+\mu\right) A_{0}\right)\right] \frac{t^{2}}{2}$

\section{RESULTS AND DISCUSSION}

In this section, we use maple software to plot the graph of semi-analytic solution of our model equations. Since, most of the parameters were not readily available; therefore we assumed them and obtain the rest from the papers we reviewed just for the purpose of illustration. The Table below shows the set of parameter values and the state variables which were used. The total number of the population of sample considered is 30000 . In order to support the analytical results, graphical representations showing the time graphs of different state variables are provided. 


\begin{tabular}{|c|c|c|}
\hline Parameters and State Variables & Value & Source \\
\hline$a$ & 0.30 & Assumed \\
\hline$b_{1}$ & 0.30 & Assumed \\
\hline$c$ & 1 & (Nthiiri, 2015). \\
\hline$d$ & 0.20 & Assumed \\
\hline$e$ & 0.20 & Assumed \\
\hline $\mathbf{N}$ & $\begin{array}{l}30000 \\
1.00\end{array}$ & $\begin{array}{l}\text { Silver and Delfim (2015). } \\
\text { Shah and Jyota, (2013). }\end{array}$ \\
\hline $\begin{array}{l}a_{A} \\
d_{H}\end{array}$ & 0.3 & (Nthiiri, 2015). \\
\hline$d_{H 1}$ & 0.24 & Assumed \\
\hline$d_{T}$ & 0.24 & Shah and Jyota (2013). \\
\hline$\lambda_{T}$ & 0.65 & Calculated \\
\hline$\lambda_{H}$ & 0.03 & Calculated \\
\hline$\mu$ & 0.02 & Shah and Jyota (2013). \\
\hline$\Lambda$ & 430 & Silver and Delfim (2015). \\
\hline$S_{0}$ & 16500 & Assumed \\
\hline$I_{0}$ & 9250 & Assumed \\
\hline$R_{0}$ & 3000 & Assumed \\
\hline$P_{1} O$ & 500 & Assumed \\
\hline $\mathrm{P}_{2} \mathrm{O}$ & 500 & Assumed \\
\hline $\mathbf{A}_{0}$ & 250 & Assumed \\
\hline$\delta$ & 0.03 & Shah and Jyota (2013). \\
\hline
\end{tabular}

BOLARIN, G.; OMATOLA, I.U.; AIYESIMI, Y.M. and YUSUF, A. 


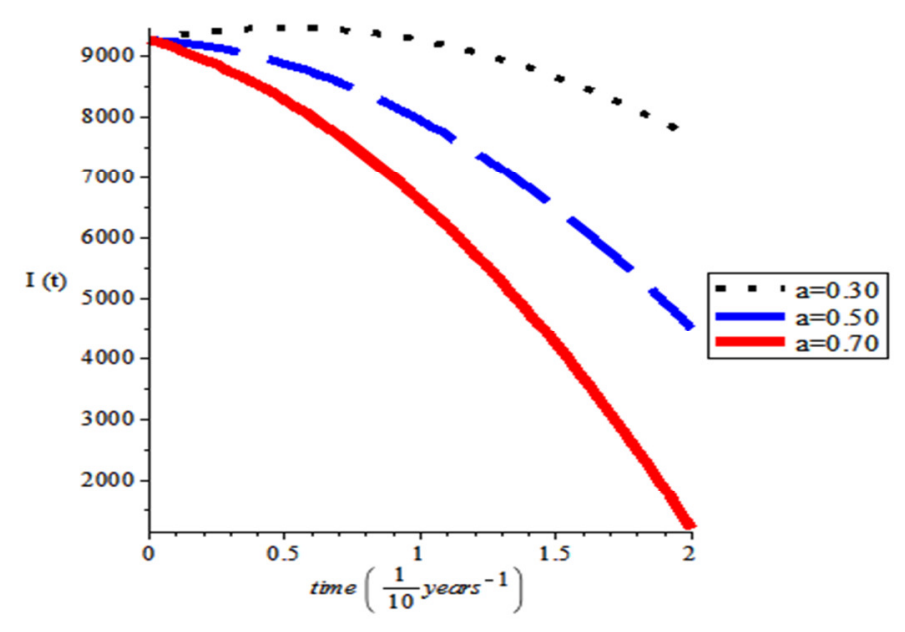

Fig 1: TB infected individuals against time

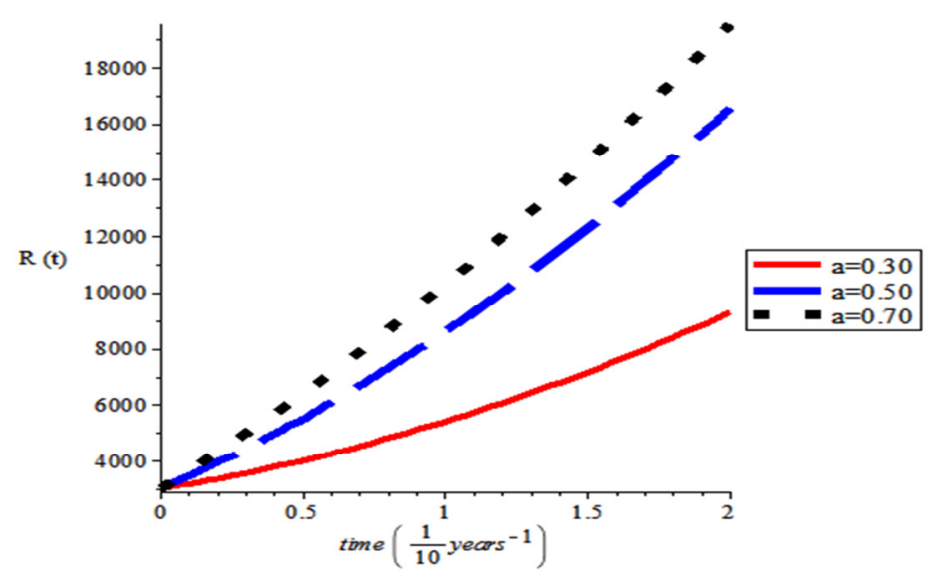

Fig 2: TB Recovered individuals against time (Low, Moderate and High treatments rate)

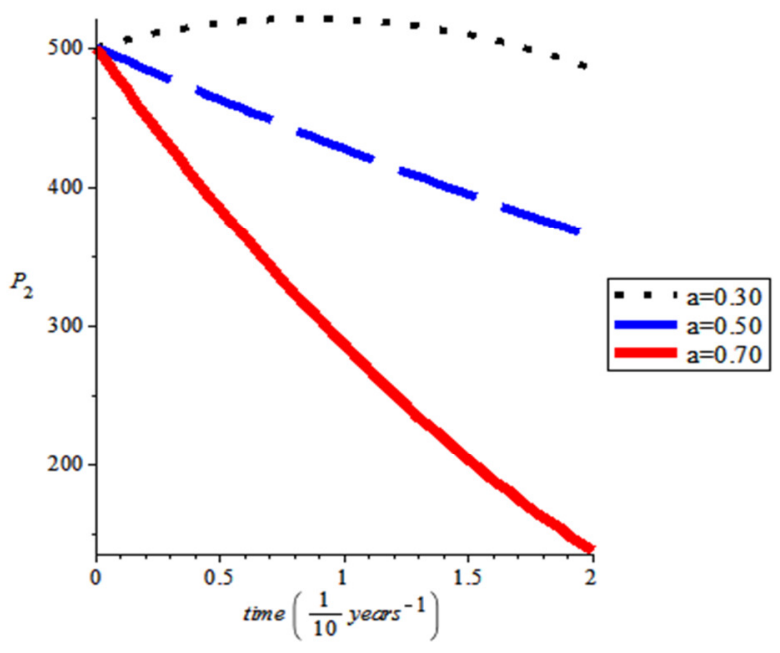

Fig 3: Low, Moderate and High treatments rate on the TB in HIV and TB co-infected individuals against time.

BOLARIN, G.; OMATOLA, I.U.; AIYESIMI, Y.M. and YUSUF, A. 


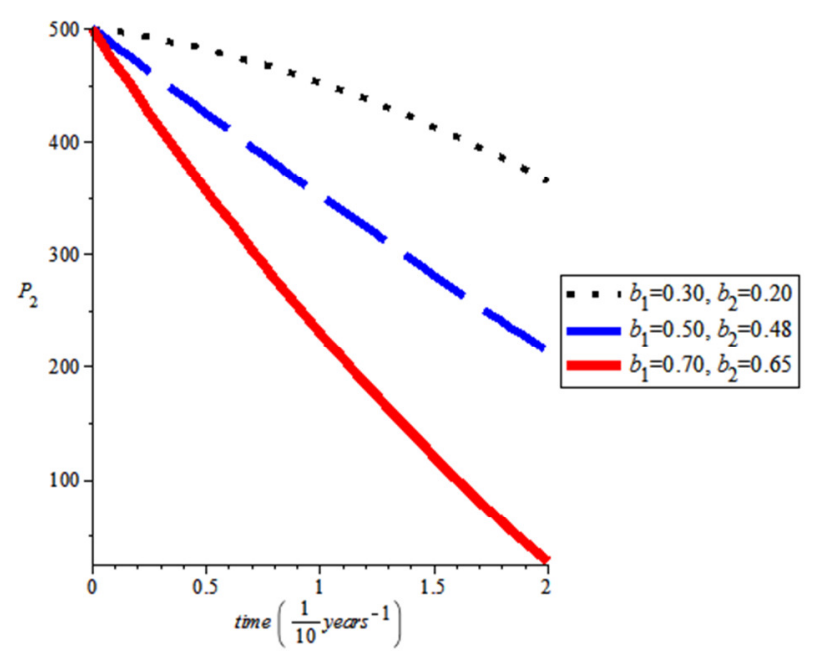

Fig 4: Co-infected individuals against time (Low, Moderate and High treatments rate)

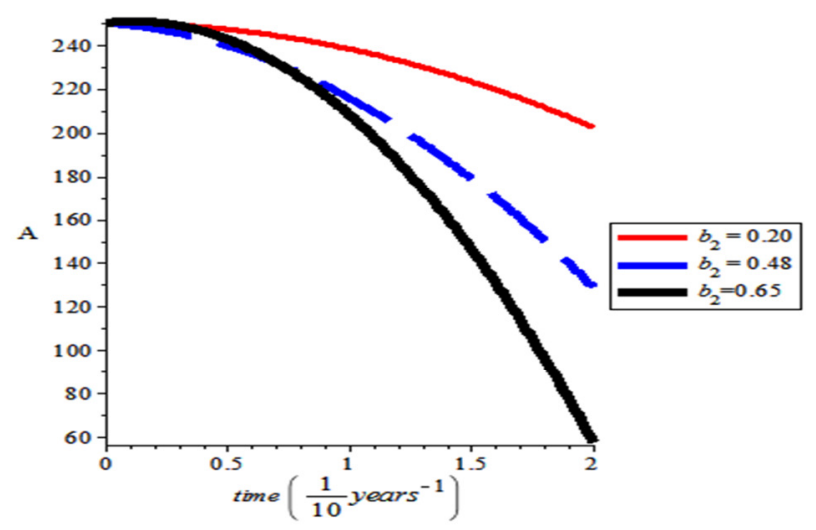

Fig 5: AIDS Infected Individuals against time for different treatment rates for HIV (Low, Moderate and High treatment rates)

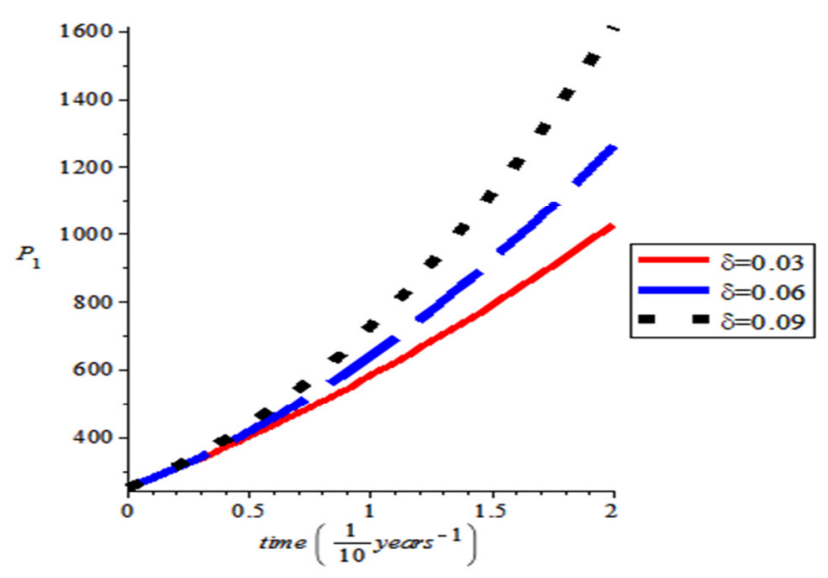

Fig 6: HIV Infected Individuals against time with different contact rates

BOLARIN, G.; OMATOLA, I.U.; AIYESIMI, Y.M. and YUSUF, A. 
Figure 1 is the graph of Infected TB individuals I (t) against time for different values of a. It is observed that the number of infected TB individuals decrease as the TB treatment parameter increases. This means, the TB can be eradicated completely in the population at time $(\mathrm{t})=20$ years when the treatment is high i.e $\mathrm{a}=0.70$.

Figure 2 displays the graph of Recovered TB individuals $\mathrm{R}(\mathrm{t})$ against time for different values of $\mathrm{a}$. It is seen that the number of TB recovered individuals increase as the TB treatment parameter a increases.

Figure 3 is the graph of TB treatment in HIV/TB coinfected individuals $\left(P_{2}\right)$ against time for different values of $b_{1}$. It is observed that the number of coinfected HIV/TB individuals decrease as the TB treatment parameter $b_{1}$ increases and brought down the number of HIV/TB co infected individuals to zero at high TB treatment rate. This means, the TB can be eradicated completely in the population at time $(\mathrm{t})$ $=20$ years.

Figure 4 is the graph of co-treatment of HIV/TB coinfected individuals against time for different values of $b_{1}$ and $b_{2}$. It is observed that the number of coinfected HIV/TB individuals decrease as the TB and HIV treatment parameters $b_{1}$ and $b_{2}$ increases and brought down the number of HIV/TB infected individuals to zero at high $\mathrm{HIV}$ and $\mathrm{TB}$ treatment rates. This means the HIV/TB co-infection can be eradicated completely in the population.

Figure 5 is the graph of AIDS Infected individuals (A) against time for different values of $b_{2}$. It is seen that the number of AIDS infected individuals decrease as the HIV treatment parameter e increases and brought down the number of individuals infected with AIDS to zero when the HIV treatment rate is high.

Figure 6 shows the graph of HIV infected individuals $\mathrm{P}_{1}$ against time for different contact rates. It is seen that the number of HIV infected individuals' increases as the HIV contact parameter $\delta$ increases.

Conclusion: The model has shown importance of TB and HIV treatments in preventing HIV and TB coinfection. It was realized that at high HIV and TB treatment rates; TB, AIDS and HIV/TB co-infection will be eradicated completely in the population. The model strongly indicated that the spread of a disease largely depend on the contact rates with infected individuals within a population. Therefore, early detection of HIV and TB cases and provision of early treatments can reduce the rate of infection, reduce the rate of progression of HIV infected individuals to AIDS and lowers co-infection.

\section{REFERENCES}

Abubakar, S; Akinwande, NI; Jimoh, OR; Oguntolu, FA; Ogwumu, OD (2013). Approximate Solution of SIR Infectious Disease Model Using Homotopy Perturbation Method (HPM)". Pacific Journal of Science and Technology. 14(2):163169.

Bolarin, G (2012). A Mathematical Model of Complacency in HIV/AIDS Scenario: SexStructure Approach, leaonardo Journal of Sciences Issue 21, P.1-12.

Bolarin, G; Omatola, IU (2016). A Mathematical Analysis of HIV/TB Co-Infection Model, Applied Mathematics. 6(4):65-72.

David, J F (2013). Mathematical Epidemiology of 
HIV/AIDS and Tuberculosis. Applied Mathematical Sciences. 9 (105):5215 - 5233.

Getahun, H; Christian, G; Reuben, G; Paul, N (2010). HIV Infection-Associated Tuberculosis: The Epidemiology and the response.

He, J.H. (1999). "Homotopy Perturbation Technique". Computer Methods in Applied Mechanics and Engineering. 178(3-4):257-262.

Ji-Haun, H. (2000). A coupling method of a homotopy technique and a perturbation technique for non-linear problems, International J. nonlinear Mech. 35:37-43.

Jiya, M. (2010). “Application of Homotopy Perturbation Method (HPM) for the Solution of some Non-Linear .Differential Equations". Pacific Journal of Science and Technology. 11(2):268-272.

Nthiiri, KJ (2015). Mathematical Modeling of
Tuberculosis as an Opportunistic Respiratory CoInfection in HIV/AIDS in the Presence of Protection. Applied Mathematical Sciences, 9(105):5215 - 5233.

Roeger, WL; Zhilan, F; Carlos, CC (2009). Mathematical Modelling of HIV/TB Coinfection. Mathematical Biosciences and Engineering, 6(4):815-837.

Shah, N. H., \& Jyota, G. (2013). Modeling of HIVTB Co infection Transmission Dynamics. American Journal of Epidemiological and Infection Disease, 2(1):1-7

Silver, J C; Delfim, F M (2015). A mathematical modeling of TB-HIV/AIDS co infection and optimal control. Discrete and Continuous Dynamical Systems. 6(7):1553-5231.

World Health Organization (2010). Antiretroviral therapy for HIV for infection in adults and adolescents. Recommendations for a public health approach: 2010 revision. Geneva 